\title{
Nordhaus-Gaddum-Type Relations for Arithmetic-Geometric Spectral Radius and Energy
}

\author{
Yajing Wang ${ }^{1}$ and Yubin Gao $\mathbb{D}^{2}$ \\ ${ }^{1}$ School of Data Science and Technology, North University of China, Taiyuan, Shanxi 030051, China \\ ${ }^{2}$ Department of Mathematics, North University of China, Taiyuan, Shanxi 030051, China \\ Correspondence should be addressed to Yubin Gao; ybgao@nuc.edu.cn
}

Received 24 February 2020; Accepted 23 June 2020; Published 16 July 2020

Academic Editor: Jean Jacques Loiseau

Copyright (c) 2020 Yajing Wang and Yubin Gao. This is an open access article distributed under the Creative Commons Attribution License, which permits unrestricted use, distribution, and reproduction in any medium, provided the original work is properly cited.

\begin{abstract}
Spectral graph theory plays an important role in engineering. Let $G$ be a simple graph of order $n$ with vertex set $V=\left\{v_{1}, v_{2}, \ldots, v_{n}\right\}$. For $v_{i} \in V$, the degree of the vertex $v_{i}$, denoted by $d_{i}$, is the number of the vertices adjacent to $v_{j}$. The arithmetic-geometric adjacency matrix $A_{a g}(G)$ of $G$ is defined as the $n \times n$ matrix whose $(i, j)$ entry is equal to $\left(\left(d_{i}+d_{j}\right) / 2 \sqrt{d_{i} d_{j}}\right)$ if the vertices $v_{i}$ and $v_{j}$ are adjacent and 0 otherwise. The arithmetic-geometric spectral radius and arithmetic-geometric energy of $G$ are the spectral radius and energy of its arithmetic-geometric adjacency matrix, respectively. In this paper, some new upper bounds on arithmeticgeometric energy are obtained. In addition, we present the Nordhaus-Gaddum-type relations for arithmetic-geometric spectral radius and arithmetic-geometric energy and characterize corresponding extremal graphs.
\end{abstract}

\section{Introduction}

Spectral properties of graphs have been widely applied in the field of engineering technology. In systems engineering, complex networks are often used as abstract models to study and reveal the relationship among system elements. The mathematical study of complex network depends on graph theory. In network dynamics, the analysis of threshold in the epidemic model and synchronization condition of coupling oscillators is essentially the study of spectral of the corresponding graph. Naturally, the development of systems engineering depends on the results and conclusions of basic studies in graph spectral theory. Similarly, in the chemical engineering, a chemical molecule can also be abstracted as a graph. The combinatorial forms of spectral of weighted graphs, based on topological indices, are closely related to the molecular orbital energy levels of $\pi$-electrons in conjugated hydrocarbons, as well as the spectrum properties. The combinatorial form of the spectrum, significantly correlated with the stability of conjugated alternant molecules, is defined as the sum of the absolute values of the eigenvalues of graphs. In this paper, we further study the arithmeticgeometric spectral radius and arithmetic-geometric energy.
Let $G=(V, E)$ be a simple graph with vertex set $V=$ $\left\{v_{1}, v_{2}, \ldots, v_{n}\right\}$ and edge set $E$, and let $|E|=m$. An edge $e \in E$ with end vertices $v_{i}$ and $v_{j}$ is denoted by $v_{i} v_{j}$. The degree of the vertex $v_{i} \in V$ of $G$ for $i=1,2, \ldots, n$ is denoted by $d_{i}$. Let $\Delta$ and $\delta$ represent the maximum and minimum degrees of $G$, respectively. The graph $\bar{G}$ is called the complement to the graph G, if its vertex set is the same as $V$, and $v_{i}$ and $v_{j}(i, j=$ $1,2, \ldots, n, i \neq j$ ) are adjacent in $\bar{G}$ if and only if they are not adjacent in $G$.

The adjacency matrix of a graph $G$ is the matrix $\left(a_{i j}\right)_{n \times n}$, denoted by $A(G)$, where $a_{i j}=1$ if $v_{i}$ and $v_{j}$ are adjacent; if not, $a_{i j}=0$. All eigenvalues of $A(G)$ are denoted by the nonincreasing sequence $\lambda_{1}, \lambda_{2}, \ldots, \lambda_{n}$. The spectral radius of $G$ is the greatest eigenvalue $\lambda_{1}$. The energy of $G$ is defined as

$$
\mathscr{E}(G)=\sum_{i=1}^{n}\left|\lambda_{i}(G)\right|
$$

As a graph invariant, topological indices are used to understand physicochemical properties of chemical compounds since they capture some properties of molecules. In 2015, the paper [1] proposed the arithmetic-geometric index of a graph $G$ and defined the arithmetic-geometric adjacency 
matrix (AG matrix) of $G$, denoted by $A_{a g}(G)$. An element of AG matrix is defined in the following manner:

$$
g_{i j}= \begin{cases}\frac{d_{i}+d_{j}}{2 \sqrt{d_{i} d_{j}},} & \text { if } v_{i} v_{j} \in E, \\ 0, & \text { otherwise. }\end{cases}
$$

The AG eigenvalues of $G$ are the eigenvalues of its corresponding AG matrix. Note that AG matrix is real and symmetric so that all eigenvalues of $A_{a g}(G)$ are real, which can be recorded as $\eta_{1} \geq \eta_{2} \geq \cdots \geq \eta_{n}$. Similarly, the greatest AG eigenvalue $\eta_{1}$ is called the arithmetic-geometric spectral radius (AG spectral radius) of $G$. In addition, the arithmeticgeometric energy (AG energy) of $G$ is defined in an analogue way as

$$
\mathscr{E}_{a g}(G)=\sum_{i=1}^{n}\left|\eta_{i}\right|
$$

The first Zagreb index $M_{1}(G)$ is a kind of important topological indices, which is defined in [2] as

$$
M_{1}(G)=\sum_{i=1}^{n} d_{i}^{2}=\sum_{v_{i} v_{j} \in E(G)}\left(d_{i}+d_{j}\right)
$$

A graph $G$ is said to be strongly regular with parameters $(n, r, \mu, v)$ if it is $r$-regular, every pair of adjacent vertices has $\mu \geq 0$ common neighbors, and every pair of distinct nonadjacent vertices has $v \geq 0$ common neighbors [3]. If $v=0$, then $G$ is a disjoint union of complete graphs, whereas if $\nu \geq 1$ and $G$ is noncomplete, then the eigenvalues of $G$ are $r$ (the trivial eigenvalue) and the roots $x_{1}$ and $x_{2}$ (the nontrivial eigenvalues) of the quadratic equation:

$$
x^{2}+(\nu-\mu) x+(\nu-r)=0 .
$$

It is straightforward to show that the complement of a strong regular graph with parameters $(n, r, \mu, \nu)$ is still a strong regular graph with parameters $(n, n-1-r$, $n-2-2 r+\nu, n-2 r+\mu)$. Recently, some bounds on AG spectral radius and AG energy were obtained in a couple of papers $[4,5]$. Until now, hundreds of such "energy" have been introduced, such as inverse sum index energy [6], distance energy $[7,8], \mathrm{ABC}$ energy $[9,10]$, matching energy $[11,12]$, and Randić energy [13, 14]. Recently, there are many studies, such as [15-17], on the energy of graphs with given parameters.

In the paper of Nordhaus and Gaddum [18], the lower and upper bounds on $\chi(G)+\chi(\bar{G})$ and $\chi(G) \cdot \chi(\bar{G})$ were given, where $\chi(G)$ and $\chi(\bar{G})$ were the chromatic number of a graph $G$ and its complement $\bar{G}$, separately. Since then, in terms of an invariant $\psi(G)$ of graph $G$, any bound on $\psi(G)+\psi(\bar{G})$ or $\psi(G) \cdot \psi(\bar{G})$ is referred to as a Nordhaus-Gaddum-type inequality or relation. And Nordhaus-Gaddum-type relations have received wide attention (e.g., [19]).

We write $G_{1} \cong G_{2}$ if the graphs $G_{1}$ and $G_{2}$ are isomorphic. As usual, let $K_{n}$ denote the complete graph of order $n$ and $K\left(p_{1}, p_{2}, \ldots, p_{n}\right)$ the complete multipartite graph, having $p_{i}$ vertices in the $i$ th partite set for each $i=1,2, \ldots, n$. Thus, $K_{n} \cong K\left(p_{1}, p_{2}, \ldots, p_{n}\right)$, where $p_{1}=p_{2}=\cdots=p_{n}=1$. For other undefined notions and terminologies from graph theory, the readers are referred to $[20,21]$. The rest of the paper is structured as follows: in Section 2, we give some useful lemmas; in Section 3, we get some new bounds on the AG energy; and in Sections 4 and 5, we obtain the Nordhaus-Gaddum-type relations for the AG spectral radius and AG energy of graph $G$, respectively.

\section{Preliminaries}

Lemma 1 (see [22]). If $B$ is an $n \times n$ real symmetric matrix with eigenvalues $\lambda_{1} \geq \lambda_{2} \geq \cdots \geq \lambda_{n}$, then for any $0 \neq \mathbf{x} \in \mathbf{R}^{n}$, $\mathbf{x}^{T} B \mathbf{x} \leq \lambda_{1} \mathbf{x}^{T} \mathbf{x}$. Equality holds if and only if $\mathbf{x}$ is an eigenvector of $B$ corresponding to $\lambda_{1}$.

Lemma 2 (see [23]). Let $G$ be a graph of order $n$ and size $m$ with maximum degree $\Delta$ and minimum degree $\delta \geq 1$. Then,

$$
\lambda_{1} \leq \sqrt{2 m-\delta(n-1)+(\delta-1) \Delta},
$$

with equality holding if and only if $G$ is regular, a star plus copies of $K_{2}$, or a complete graph plus a regular graph with a smaller degree of vertices.

Lemma 3 (see [5]). Let $G$ be a graph of order $n$ with the maximum degree $\Delta$ and minimum degree $\delta \geq 1$. Then,

$$
\eta_{1} \leq \frac{1}{2}\left(\sqrt{\frac{\Delta}{\delta}}+\sqrt{\frac{\delta}{\Delta}}\right) \lambda_{1}
$$

with equality holding if and only if $G$ is regular.

Lemma 4 (see [5]). Let $G$ be a graph of order $n$. Then,

$$
\eta_{1} \geq \sqrt{\frac{M_{1}(G)}{n}}
$$

with equality holding if and only if $G$ is regular.

Lemma 5 (see [24]). A connected graph $G$ of order $n$ has only one positive eigenvalue in its adjacency spectrum if and only if $G$ is a complete multipartite graph.

\section{On AG Energy of a Graph}

Lemma 6. Let $G$ be a graph of order $n$ and size $m$, then

$$
\eta_{1} \geq \frac{2 m}{n}
$$

equality holds if and only if $G$ is a regular graph.

Proof. Let us take any unit vector $\mathbf{x}=\left(x_{1}, x_{2}, \ldots, x_{n}\right)^{T}$ in $\mathbf{R}^{n}$. By Lemma 1, we have

$$
\eta_{1} \geq \mathbf{x}^{T} A_{a g}(G) \mathbf{x}=\sum_{v_{i} v_{j} \in E(G)} \frac{d_{i}+d_{j}}{\sqrt{d_{i} d_{j}}} x_{i} x_{j} \geq 2 \sum_{v_{i} v_{j} \in E(G)} x_{i} x_{j} .
$$



have

Taking $\mathbf{x}=((1 / \sqrt{n}),(1 / \sqrt{n}), \ldots,(1 / \sqrt{n}))^{T}$ into (10), we

$$
\eta_{1}(G) \geq \mathbf{x}^{T} A_{a g}(G) \mathbf{x} \geq \frac{2 m}{n},
$$

then (9) holds.

For the equality in (9) to hold, all the inequalities in the above argument must be equalities. From (10), we have $d_{1}=d_{2}=\cdots=d_{n}$. Then, $G$ is a regular graph.

Conversely, if $G$ is regular, then $d_{1}=d_{2}=\cdots=d_{n}$. So $\mathbf{x}=((1 / \sqrt{n}),(1 / \sqrt{n}), \ldots,(1 / \sqrt{n}))^{T}$ is an eigenvector of $A_{a g}(G)$ corresponding to the eigenvalue $\eta_{1}$. Then, the equality holds in (9).

Theorem 1. Let $G$ be a graph of order $n$ and size $m$, then

$$
\mathscr{E}_{\text {ag }}(G) \geq \frac{4 m}{n},
$$

with equality holding if and only if $G$ is isomorphic to a regular complete multipartite graph or $G \cong \bar{K}_{n}$.

Proof. Applying Lemma 6, we have

$$
\mathscr{E}_{\text {ag }}(G)=\sum_{i=1}^{n}\left|\eta_{i}\right|=2 \sum_{i=1, \eta_{i} \geq 0}^{n} \eta_{i} \geq 2 \eta_{1} \geq \frac{4 m}{n} .
$$

The first equality attained if and only if $G$ has at most one positive $A G$ eigenvalue, and the second equality holds if and only if $G$ is regular. If $G$ has no positive eigenvalue, then $G \cong \bar{K}_{n}$. If $G$ has only one positive eigenvalue, from Lemma 5 , then $G$ is isomorphic to a complete multipartite graph. And, if $G$ is a regular graph, then its AG matrix is identical to its adjacency matrix. If so, we know that the equality in Theorem 1 holds if and only if $G$ is isomorphic to a regular complete multipartite graph, or $G \cong \bar{K}_{n}$.

Theorem 2. Let $G$ be a graph of order $n$ and size $m$, and $\delta>0$. Then,

$$
\mathscr{E}_{a g}(G) \leq t+\sqrt{(n-1)\left[\frac{m}{2}\left(\sqrt{\frac{\Delta}{\delta}}+\sqrt{\frac{\delta}{\Delta}}\right)^{2}-t^{2}\right]},
$$

where $t=\max \{(2 m / n),(\sqrt{\Delta / \delta}+\sqrt{\delta / \Delta}) \sqrt{m / 2 n}\}$. This bound is achieved if $G$ is either $(n / 2) K_{2}, K_{n}$, or a noncomplete connected strongly regular graph with two nontrivial eigenvalues both with absolute value:

$$
\sqrt{\frac{\left[2 m-(2 m / n)^{2}\right]}{n-1}} \text {. }
$$

Proof. By Lemma 6, we have $\eta_{1} \geq(2 m / n)$. Moreover, since

$$
\begin{aligned}
\sum_{i=1}^{n} \eta_{i}^{2} & =\frac{1}{2} \sum_{v_{i} v_{j} \in E(G)}\left(\frac{d_{i}+d_{j}}{\sqrt{d_{i} d_{j}}}\right)^{2} \\
& =\frac{1}{2} \sum_{v_{i} v_{j} \in E(G)}\left(\sqrt{\frac{d_{i}}{d_{j}}}+\sqrt{\frac{d_{j}}{d_{i}}}\right)^{2} \\
& \leq \frac{1}{2} \sum_{v_{i} v_{j} \in E(G)}\left(\sqrt{\frac{\Delta}{\delta}}+\sqrt{\frac{\delta}{\Delta}}\right)^{2} \\
& =\frac{m}{2}\left(\sqrt{\frac{\Delta}{\delta}}+\sqrt{\frac{\delta}{\Delta}}\right)^{2}
\end{aligned}
$$

must hold, we have

$$
\sum_{i=2}^{n} \eta_{i}^{2} \leq \frac{m}{2}\left(\sqrt{\frac{\Delta}{\delta}}+\sqrt{\frac{\delta}{\Delta}}\right)^{2}-\eta_{1}^{2}
$$

Combining the above inequality with the Cauchy-Schwartz inequality, we can see that the following inequality is obvious:

$$
\sum_{i=2}^{n}\left|\eta_{i}\right| \leq \sqrt{(n-1)\left[\frac{m}{2}\left(\sqrt{\frac{\Delta}{\delta}}+\sqrt{\frac{\delta}{\Delta}}\right)^{2}-\eta_{1}^{2}\right]} .
$$

Thus,

$$
\mathscr{E}_{a g}(G) \leq \eta_{1}+\sqrt{(n-1)\left[\frac{m}{2}\left(\sqrt{\frac{\Delta}{\delta}}+\sqrt{\frac{\delta}{\Delta}}\right)^{2}-\eta_{1}^{2}\right]} .
$$

Since the function

$$
F(x)=x+\sqrt{(n-1)\left[\frac{m}{2}\left(\sqrt{\frac{\Delta}{\delta}}+\sqrt{\frac{\delta}{\Delta}}\right)^{2}-x^{2}\right]}
$$

reaches the maximum for $x=(\sqrt{\Delta / \delta}+\sqrt{\delta / \Delta}) \sqrt{m / 2 n}$, we see that $F\left(\eta_{1}\right) \leq F((\sqrt{\Delta / \delta}+\sqrt{\delta / \Delta}) \sqrt{m / 2 n})$ must hold as well. In addition, if $(2 m / n) \geq(\sqrt{\Delta / \delta}+\sqrt{\delta / \Delta}) \sqrt{m / 2 n}$, then $F\left(\eta_{1}\right) \leq F(2 m / n)$. From this fact and inequality (19), it immediately follows that inequality (14) holds when $t=\max \{(2 m / n),(\sqrt{\Delta / \delta}+\sqrt{\delta / \Delta}) \sqrt{m / 2 n}\}$.

It is obvious that $2 m \geq n$ and we know that if $G$ is either $(n / 2) K_{2}, K_{n}$, or a noncomplete connected strongly regular graph, the inequality $(2 m / n) \geq(\sqrt{\Delta / \delta}+\sqrt{\delta / \Delta}) \sqrt{m / 2 n}$ holds. As the graph $(n / 2) K_{2}$ is regular, its AG matrix is the same as its adjacency matrix. That is to say, the AG eigenvalues for $(n / 2) K_{2}$ are \pm 1 (both with multiplicity $(n / 2))$. Similarly, the AG eigenvalues for $K_{n}$ are $n-1$ (multiplicity 1 ) and -1 (multiplicity $n-1$ ) so that equality must hold in (14), if $G$ is isomorphic to $(n / 2) K_{2}$ or $K_{n}$.

Moreover, if $G$ is a noncomplete connected strongly regular graph with the trivial eigenvalue $(2 \mathrm{~m} / \mathrm{n})$ and other two nontrivial eigenvalues both with absolute value $\sqrt{\left[2 m-(2 m / n)^{2}\right] /(n-1)}$, the equality holds in (14) as well. 
Remark 1. The paper [4] proved that if $G$ is a simple graph of order $n$ and size $m$ having no isolated vertices, then the following bound must hold:

$$
\mathscr{E}_{a g}(G) \leq \frac{\Delta}{\delta} \sqrt{2 m n} .
$$

The reason is following: $F(x)$ defined in the proof of Theorem 2 reaches the maximum for $x=(\sqrt{\Delta / \delta}+$ $\sqrt{\delta / \Delta}) \sqrt{m / 2 n}$, and the maximum is $F(x)=(1 / 2)(\sqrt{\Delta / \delta}+$ $\sqrt{\delta / \Delta}) \sqrt{2 m n}$. It follows that

$$
\mathscr{E}_{\text {ag }}(G) \leq \frac{1}{2}\left(\sqrt{\frac{\Delta}{\delta}}+\sqrt{\frac{\delta}{\Delta}}\right) \sqrt{2 m n}
$$

must hold. Moreover, $(1 / 2)(\sqrt{\Delta / \delta}+\sqrt{\delta / \Delta}) \sqrt{2 m n} \leq$ $(\Delta / \delta) \sqrt{2 m n}$. Hence, inequality (14) is an improvement on the bound in [4].

Theorem 3. If $2 m \leq n$ and $G$ is a graph on $n$ vertices with $m$ edges, then

$$
\mathscr{E}_{a g}(G) \leq m\left(\sqrt{\frac{\Delta(\Gamma)}{\delta(\Gamma)}}+\sqrt{\frac{\delta(\Gamma)}{\Delta(\Gamma)}}\right),
$$

where $\Gamma$ is the graph obtained from $G$ by deleting all isolated vertices. Equality holds if and only if $\Gamma \cong(n / 2) K_{2}$.

Proof. It is easy to know that $G$ has at least $n-2 m$ isolated vertices if $2 m \leq n$. We can obtain a graph $\Gamma$ with no isolated vertices by removing all isolated vertices. It follows that $\Gamma$ has at most $2 m$ vertices. Hence, by applying inequality (22) to $\Gamma$, we can immediately see that

$$
\mathscr{E}_{a g}(G) \leq \frac{1}{2}\left(\sqrt{\frac{\Delta(\Gamma)}{\delta(\Gamma)}}+\sqrt{\frac{\delta(\Gamma)}{\Delta(\Gamma)}}\right) \sqrt{2 m n} \leq m\left(\sqrt{\frac{\Delta(\Gamma)}{\delta(\Gamma)}}+\sqrt{\frac{\delta(\Gamma)}{\Delta(\Gamma)}}\right) .
$$

Moreover, equality holds if and only if $2 m=n$; that is, $G$ is the disjoint union of edges.

Theorem 4. Let $G$ be a graph of order $n$ and size $m$ and $\delta>0$. Then,

$$
\mathscr{E}_{a g}(G) \leq \frac{n+n \sqrt{n}}{8}\left(\sqrt{\frac{\Delta}{\delta}}+\sqrt{\frac{\delta}{\Delta}}\right)^{2}
$$

holds. If $G$ is a strongly regular graph with parameters $(n,((n+\sqrt{n}) / 2),((n+2 \sqrt{n}) / 4),((n+2 \sqrt{n}) / 4))$, the bound is achieved.

Proof. It is obvious that $2 m \geq n$. If $(2 m / n) \geq(\sqrt{\Delta / \delta}+$ $\sqrt{\delta / \Delta}) \sqrt{m / 2 n}$, then the inequality (14) is as follows:

$$
\mathscr{E}_{a g}(G) \leq \frac{2 m}{n}+\sqrt{(n-1)\left[\frac{m}{2}\left(\sqrt{\frac{\Delta}{\delta}}+\sqrt{\frac{\delta}{\Delta}}\right)^{2}-\left(\frac{2 m}{n}\right)^{2}\right]} .
$$

Using routine calculus, it is seen that the left-hand side of inequality (26) - considered as a function of $m$-is maximized when

$$
m=\frac{n^{2}+n \sqrt{n}}{16}\left(\sqrt{\frac{\Delta}{\delta}}+\sqrt{\frac{\delta}{\Delta}}\right)^{2},
$$

holds. Inequality (25) now follows by substituting this value of $m$ into (26).

If $(\sqrt{\Delta / \delta}+\sqrt{\delta / \Delta}) \sqrt{m / 2 n} \geq(2 m / n)$, then by inequality (22) and $2 m \geq n$, we know that

$$
\mathscr{E}_{a g}(G) \leq \frac{n}{4}\left(\sqrt{\frac{\Delta}{\delta}}+\sqrt{\frac{\delta}{\Delta}}\right)^{2} .
$$

Since

$$
((n+n \sqrt{n}) / 8)(\sqrt{\Delta / \delta}+\sqrt{\delta / \Delta})^{2}>
$$
$(n / 4)(\sqrt{\Delta / \delta}+\sqrt{\delta / \Delta})^{2}$, then it is clear that (25) follows. Moreover, it follows by Theorem 2 that if $G$ is a strongly regular graph with parameters $(n,((n+\sqrt{n}) / 2)$, $((n+2 \sqrt{n}) / 4),((n+2 \sqrt{n}) / 4))$, equality holds in (25), which means that the upper bound is achieved. The theorem follows.

\section{Nordhaus-Gaddum-Type Relation for AG Spectral Radius}

Let $\bar{\Delta}, \bar{\delta}$, and $\bar{\eta}_{1}$ be the maximum degree, minimum degree, and spectral radius of the complement graph $\bar{G}$ of $G$, respectively. Here, we are presenting the lower and upper bounds on $\eta_{1}+\overline{\eta_{1}}$.

Theorem 5. Let $G$ be a graph of order $n$. Then,

$$
\eta_{1}+\bar{\eta}_{1} \geq \sqrt{\frac{M_{1}(G)}{n}}+\sqrt{\frac{M_{1}(\bar{G})}{n}}
$$

and the equality holds if and only if $G$ is a regular graph.

Proof. The proof of inequality in (29) follows directly from Lemma 4.

If the equality in (29) holds, that is, $\eta_{1}=\sqrt{M_{1}(G) / n}$ and $\bar{\eta}_{1}=\sqrt{M_{1}(\bar{G}) / n}$, then by Lemma $4, G$ is regular.

If $G$ is a regular graph, then

$$
\eta_{1}+\bar{\eta}_{1}=\Delta+n-1-\delta=n-1=\sqrt{\frac{M_{1}(G)}{n}}+\sqrt{\frac{M_{1}(\bar{G})}{n}} .
$$

This finishes the proof.

Theorem 6. Let $G$ be a graph of order $n$. Then,

$$
\eta_{1}+\bar{\eta}_{1} \geq n-1
$$

with equality holding if and only if $G$ is a regular graph.

Proof. Lemma 6 gives that 


$$
\begin{aligned}
& \eta_{1} \geq \frac{2 m}{n}, \\
& \bar{\eta}_{1} \geq \frac{2|E(\bar{G})|}{n},
\end{aligned}
$$

where $|E(\bar{G})|$ denotes the number of edges in $\bar{G}$. Then, we have

$$
\eta_{1}+\bar{\eta}_{1} \geq \frac{2 m}{n}+\frac{2|E(\bar{G})|}{n}=\frac{2}{n}\left(\begin{array}{l}
n \\
2
\end{array}\right)=n-1 .
$$

From Lemma 6, equality holds if and only if $G$ is isomorphic to a regular graph.

Theorem 7. Let $G$ be a connected graph of order $n$ and size $m$. And we write the number of vertices with $d_{i}=\Delta$ by $t$ and the second largest degree of graph $G$ by $\tau$ :

(1) If $\Delta=n-1$ or $\bar{\Delta}=n-1$, then

$$
\begin{aligned}
\eta_{1}+\bar{\eta}_{1} \leq & \frac{1}{2}\left(\sqrt{\frac{n-1}{\delta}}+\sqrt{\frac{\delta}{n-1}}\right) \sqrt{2 m-n+1} \\
& +\frac{1}{2}\left(\sqrt{\frac{n-t-1}{n-1-\tau}}+\sqrt{\frac{n-1-\tau}{n-t-1}}\right) \\
& \cdot \sqrt{(n-1)^{2}-2 m+t} .
\end{aligned}
$$

(2) If $\Delta \leq n-2$ and $\bar{\Delta} \leq n-2$, then

$$
\begin{aligned}
\eta_{1}+\bar{\eta}_{1} \leq & \frac{1}{2}\left(\sqrt{\frac{\Delta}{\delta}}+\sqrt{\frac{\delta}{\Delta}}\right) \sqrt{2 m-\delta(n-1)+(\delta-1) \Delta} \\
& +\frac{1}{2}\left(\sqrt{\frac{n-1-\delta}{n-1-\Delta}}+\sqrt{\frac{n-1-\Delta}{n-1-\delta}}\right) \\
& \cdot \sqrt{(n-1)^{2}-2 m-\delta(n-2-\Delta)} .
\end{aligned}
$$

Moreover, the bounds are sharp.

Proof. (1) Without loss of generality, let $\Delta=n-1$. Since $\delta \geq 1$, by Lemmas 2 and 3 , we have

$$
\begin{aligned}
\eta_{1} & \leq \frac{1}{2}\left(\sqrt{\frac{n-1}{\delta}}+\sqrt{\frac{\delta}{n-1}}\right) \sqrt{2 m-\delta(n-1)+(\delta-1)(n-1)} \\
& =\frac{1}{2}\left(\sqrt{\frac{n-1}{\delta}}+\sqrt{\frac{\delta}{n-1}}\right) \sqrt{2 m-n+1} .
\end{aligned}
$$

It clearly follows that $\bar{G}$ has $t$ isolated vertices. Let $\Gamma_{1}$ be the graph obtained from $\bar{G}$ by removing the isolated vertices. It is clear that $\bar{\eta}_{1}=\eta_{1}\left(\Gamma_{1}\right), \bar{\Delta}=\Delta\left(\Gamma_{1}\right) \leq n-t-1$, and $\bar{\delta}=\delta\left(\Gamma_{1}\right)=n-1-\tau$, so

$$
\begin{aligned}
\bar{\eta}_{1} \leq & \frac{1}{2}\left(\sqrt{\frac{\Delta\left(\Gamma_{1}\right)}{\delta\left(\Gamma_{1}\right)}}+\sqrt{\frac{\delta\left(\Gamma_{1}\right)}{\Delta\left(\Gamma_{1}\right)}}\right) \\
& \cdot \sqrt{2 e\left(\Gamma_{1}\right)-\delta\left(\Gamma_{1}\right)\left(\left|V\left(\Gamma_{1}\right)\right|-1\right)+\left(\delta\left(\Gamma_{1}\right)-1\right) \Delta\left(\Gamma_{1}\right)} \\
\leq & \frac{1}{2}\left(\sqrt{\frac{n-t-1}{n-1-\tau}}+\sqrt{\frac{n-1-\tau}{n-t-1}}\right) \sqrt{2\left(\begin{array}{l}
n \\
2
\end{array}\right)-2 m-(n-t-1)} \\
= & \frac{1}{2}\left(\sqrt{\frac{n-t-1}{n-1-\tau}}+\sqrt{\frac{n-1-\tau}{n-t-1}}\right) \sqrt{(n-1)^{2}-2 m+t} .
\end{aligned}
$$

The proof of the inequality (34) follows.

(2) Let $\Delta \leq n-2$ and $\bar{\Delta} \leq n-2$. It follows that $\delta \geq 1$ and $\bar{\delta} \geq 1$. From Lemmas 2 and 3, we have

$$
\begin{aligned}
\eta_{1} \leq & \frac{1}{2}\left(\sqrt{\frac{\Delta}{\delta}}+\sqrt{\frac{\delta}{\Delta}}\right) \sqrt{2 m-\delta(n-1)+(\delta-1) \Delta} \\
\bar{\eta}_{1} \leq & \frac{1}{2}\left(\sqrt{\frac{\bar{\Delta}}{\bar{\delta}}}+\sqrt{\frac{\bar{\delta}}{\bar{\Delta}}}\right) \sqrt{2\left(\begin{array}{c}
n \\
2
\end{array}\right)-2 m-\bar{\delta}(n-1)+(\bar{\delta}-1) \bar{\Delta}} \\
= & \frac{1}{2}\left(\sqrt{\frac{n-1-\delta}{n-1-\Delta}}+\sqrt{\frac{n-1-\Delta}{n-1-\delta}}\right) \\
& \cdot \sqrt{2\left(\begin{array}{l}
n \\
2
\end{array}\right)-2 m-(n-1-\Delta)(n-1)+(n-2-\Delta)(n-1-\delta)} \\
= & \frac{1}{2}\left(\sqrt{\frac{n-1-\delta}{n-1-\Delta}}+\sqrt{\frac{n-1-\Delta}{n-1-\delta}}\right) \sqrt{(n-1)^{2}-2 m-\delta(n-2-\Delta)} .
\end{aligned}
$$

Hence, the inequality (35) holds.

To show the sharpness of upper bounds in Theorem 7 , we consider the following examples.

Example 1. Let $G$ be a star of order $n$. That is to say, $t=1$ and $\tau=1$. Then, $\bar{G}$ is the union of an isolated vertex and a complete graph $K_{n-1}$. We have

$$
\begin{aligned}
\eta_{1}= & \frac{n}{2}=\frac{1}{2}\left(\sqrt{n-1}+\frac{1}{\sqrt{n-1}}\right) \sqrt{n-1} \\
= & \frac{1}{2}\left(\sqrt{\frac{n-1}{\delta}}+\sqrt{\frac{\delta}{n-1}}\right) \sqrt{2 m-n+1} \\
\bar{\eta}_{1}= & n-2=\frac{1}{2}\left(\sqrt{\frac{n-t-1}{n-1-\tau}}+\sqrt{\frac{n-1-\tau}{n-t-1}}\right) \\
& \cdot \sqrt{(n-1)^{2}-2 m+t} .
\end{aligned}
$$

Example 2. Let $G$ be a regular graph. It is clear that $\bar{G}$ is still a regular graph. We have 


$$
\begin{aligned}
\eta_{1}+\bar{\eta}_{1}= & \Delta+(n-1-\Delta)=n-1 \\
= & \frac{1}{2}\left(\sqrt{\frac{\Delta}{\delta}}+\sqrt{\frac{\delta}{\Delta}}\right) \sqrt{2 m-\delta(n-1)+(\delta-1) \Delta} \\
& +\frac{1}{2}\left(\sqrt{\frac{n-1-\delta}{n-1-\Delta}}+\sqrt{\frac{n-1-\Delta}{n-1-\delta}}\right) \\
& \cdot \sqrt{(n-1)^{2}-2 m-\delta(n-2-\Delta)} .
\end{aligned}
$$

\section{Nordhaus-Gaddum-Type Relation for AG Energy}

In the following theorems, some lower and upper bounds for $\mathscr{E}_{a g}(G)+\mathscr{E}_{a g}(\bar{G})$ are obtained.

Theorem 8. Let $G$ be a connected graph of order $n$ and size $m$ and $\Gamma_{1}, \Gamma_{2}, \ldots, \Gamma_{r}$ be all connected components of $\bar{G}$. Then,

$$
\mathscr{E}_{a g}(G)+\mathscr{E}_{a g}(\bar{G}) \geq \frac{4 m}{n}+\sum_{i=1}^{r} \frac{4\left|E\left(\Gamma_{i}\right)\right|}{\left|V\left(\Gamma_{i}\right)\right|}
$$

where $\left|V\left(\Gamma_{i}\right)\right|$ and $\left|E\left(\Gamma_{i}\right)\right|$ are the number of vertices and edges in $\Gamma_{i}(i=1, \ldots, r)$, respectively. Moreover, the equality holds if and only if $G$ is isomorphic to a regular complete multipartite graph, or $G \cong \bar{K}_{n}$.

Proof. Applying Theorem 1, we have

$$
\begin{aligned}
& \mathscr{E}_{a g}(G) \geq \frac{4 m}{n}, \\
& \mathscr{E}_{a g}\left(\Gamma_{i}\right) \geq \frac{4\left|E\left(\Gamma_{i}\right)\right|}{\left|V\left(\Gamma_{i}\right)\right|} .
\end{aligned}
$$

Hence, we can obtain

$$
\mathscr{E}_{a g}(G)+\mathscr{E}_{a g}(\bar{G})=\mathscr{E}_{a g}(G)+\sum_{i=1}^{r} \mathscr{E}_{a g}\left(\Gamma_{i}\right) \geq \frac{4 m}{n}+\sum_{i=1}^{r} \frac{4\left|E\left(\Gamma_{i}\right)\right|}{\left|V\left(\Gamma_{i}\right)\right|} .
$$

From Theorem 1, we know that equality is possible if and only if $G$ is isomorphic to regular complete multipartite graph, or $G \cong \bar{K}_{n}$. The complement of regular complete multipartite graph is the disjoint union of $k$ complete graphs of order $(n / k)$, and $K_{n}$ is the complement graph of $\bar{K}_{n}$. If so, $\mathscr{E}_{\text {ag }}\left(\Gamma_{i}\right)=\left(4\left|E\left(\Gamma_{i}\right)\right| /\left|V\left(\Gamma_{i}\right)\right|\right)$ holds. Hence, the equality holds if and only if $G$ is isomorphic to a regular complete multipartite graph, or $G \cong \bar{K}_{n}$.

Theorem 9. Let $G$ be a graph of order $n$ and size $m$, then

$$
\mathscr{E}_{a g}(G)+\mathscr{E}_{a g}(\bar{G}) \geq 2(n-1),
$$

with equality holding if and only if either $G \cong K_{n}$ or $G \cong \bar{K}_{n}$.

Proof. Note that

$$
\begin{aligned}
\mathscr{E}_{a g}(G)+\mathscr{E}_{a g}(\bar{G}) & \geq \frac{4 m}{n}+\frac{4|E(\bar{G})|}{n} \\
& =\frac{4}{n}\left(\begin{array}{c}
n \\
2
\end{array}\right) \\
& =2(n-1) .
\end{aligned}
$$

Similar to the analysis of equality in Theorem 8 , we consider the following two cases:

(1) $G \cong K_{n}$, which implies $\bar{G} \cong \bar{K}_{n}$.

(2) Both $G$ and $\bar{G}$ are isomorphic to regular complete multipartite graphs. This is impossible because complete multipartite graphs are connected, whereas their complements are disconnected.

Theorem 10. Let $G$ be a graph of order $n$ and $\bar{G}$ is the complement graph of $G$. If both $G$ and $\bar{G}$ have no isolated vertices, then

$$
\mathscr{E}_{a g}(G)+\mathscr{E}_{a g}(\bar{G}) \leq \frac{n+n \sqrt{n}}{4}\left(\frac{\Delta(n-1-\delta)}{\delta(n-1-\Delta)}+3\right) .
$$

If $G$ is a strongly regular graph with parameters $(n,((n+\sqrt{n}) / 2),((n+2 \sqrt{n}) / 4),((n+2 \sqrt{n}) / 4))$, the equality holds.

Proof. From Theorem 4, we have

$$
\begin{aligned}
\mathscr{E}_{a g}(G)+\mathscr{E}_{a g}(\bar{G}) & \leq \frac{n+n \sqrt{n}}{8}\left(\sqrt{\frac{\Delta}{\delta}}+\sqrt{\frac{\delta}{\Delta}}\right)^{2}+\frac{n+n \sqrt{n}}{8}\left(\sqrt{\frac{\bar{\Delta}}{\bar{\delta}}}+\sqrt{\frac{\bar{\delta}}{\bar{\Delta}}}\right)^{2} \\
& =\frac{n+n \sqrt{n}}{8}\left[\left(\sqrt{\frac{\Delta}{\delta}}+\sqrt{\frac{\delta}{\Delta}}\right)^{2}+\left(\sqrt{\frac{n-1-\delta}{n-1-\Delta}}+\sqrt{\frac{n-1-\Delta}{n-1-\delta}}\right)^{2}\right] \\
& \leq \frac{n+n \sqrt{n}}{4}\left(\frac{\Delta(n-1-\delta)}{\delta(n-1-\Delta)}+3\right) .
\end{aligned}
$$


If $G$ is a strongly regular graph, we know that $\bar{G}$ is also a strongly regular graph. Moreover, all inequalities in the above argument must be equalities. The first equality holds if $G$ is isomorphic to a strongly regular graph with parameters $(n,((n+\sqrt{n}) / 2),((n+2 \sqrt{n}) / 4),((n+2 \sqrt{n}) / 4))$. And, the last equality holds if $G$ is regular. The theorem now follows immediately.

\section{Conclusions}

In this paper, we obtain an upper bound on AG spectral radius, and furthermore, the Nordhaus-Gaddum-type relation of AG spectral radius is derived. Some new upper and lower bounds on arithmetic-geometric energy are obtained. Moreover, we illustrate one of the upper bounds is better than the result in paper [4]. In the end, we state the Nordhaus-Gaddum-type relation of AG energy.

\section{Data Availability}

No data were used to support this study.

\section{Conflicts of Interest}

The authors declare that there are no conflicts of interest regarding the publication of this paper.

\section{Acknowledgments}

This research was funded by the Shanxi Scholarship Council of China (no. 201901D211227).

\section{References}

[1] V. Shegehalli and R. Kanabur, "Arithmetic-geometric indices of path graph," Journal of Computer and Mathematical Sciences, vol. 6, no. 1, pp. 19-24, 2015.

[2] I. Gutman and N. Trinajstić, "Graph theory and molecular orbitals. Total $\varphi$-electron energy of alternant hydrocarbons," Chemical Physics Letters, vol. 17, no. 4, pp. 535-538, 1972.

[3] C. Godsil and G. F. Royle, Algebraic Graph Theory, Springer, New York, NY, USA, 2001.

[4] X. Guo and Y. B. Gao, "Arithmetic-geometric spectral radius and energy of graphs," MATCH Communications in Mathematical and in Computer Chemistry, vol. 83, no. 3, pp. 651-660, 2020.

[5] L. Zheng, G. X. Tian, and S. Y. Cui, "On spectral radius and energy of arithmetic-geometric matrix of graphs," MATCH Communications in Mathematical and in Computer Chemistry, vol. 83, no. 3, pp. 635-650, 2020.

[6] S. Hafeez and R. Farooq, "Inverse sum indeg energy of graphs," IEEE Access, vol. 7, pp. 100860-100866, 2019.

[7] X. Zhang, "Inertia and distance energy of line graphs of unicyclic graphs," Discrete Applied Mathematics, vol. 254, pp. 222-233, 2019.

[8] S. Pirzada, H. A. Ganie, B. A. Rather, and R. Ul Shaban, "On generalized distance energy of graphs," Linear Algebra and Its Applications, vol. 603, pp. 1-19, 2020.

[9] X. Chen, "On ABC eigenvalues and ABC energy," Linear Algebra and Its Applications, vol. 544, pp. 141-157, 2018.
[10] Y. Gao and Y. Shao, "The minimum ABC energy of trees," Linear Algebra and Its Applications, vol. 577, pp. 186-203, 2019.

[11] J. Zhu and J. Yang, "On the minimal matching energies of unicyclic graphs," Discrete Applied Mathematics, vol. 254, pp. 246-255, 2019.

[12] F. Ashraf, "Energy, matching number and odd cycles of graphs," Linear Algebra and Its Applications, vol. 577, pp. 159-167, 2019.

[13] K. C. Das and S. Sun, "Extremal graphs for Randić energy," MATCH Communications in Mathematical and in Computer Chemistry, vol. 77, no. 1, pp. 77-84, 2017.

[14] I. Altındag, "Some statistical results on Randić energy of graphs," MATCH Communications in Mathematical and in Computer Chemistry, vol. 79, no. 2, pp. 331-339, 2018.

[15] M. A. Bhat and S. Pirzada, "On unicyclic graphs with given number of pendent vertices and minimal energy," Linear Algebra and Its Applications, vol. 597, pp. 86-93, 2020.

[16] H. A. Ganie, U. Samee, S. Pirzada, and A. M. Alghamdi, "Bounds for graph energy in terms of vertex covering and clique numbers," Electronic Journal of Graph Theory and Applications, vol. 7, no. 2, pp. 315-328, 2019.

[17] H. A. Ganie, U. Samee, and S. Pirzada, "On graph energy, maximum degree and vertex cover number," Le Matematiche, vol. 74, no. 1, pp. 163-172, 2019.

[18] E. A. Nordhaus and J. W. Gaddum, "On complementary graphs," The American Mathematical Monthly, vol. 63, no. 3, pp. 175-177, 1956.

[19] M. Aouchiche and P. Hansen, "A survey of NordhausGaddum type relations," Discrete Applied Mathematics, vol. 161, no. 4-5, pp. 466-546, 2013.

[20] J. A. Bondy and U. S. R. Murty, Graph Theory with Applications, Macmillan, London, UK, 1976.

[21] X. Li, Y. Shi, and I. Gutman, Graph Energy, Springer, New York, NY, USA, 2012.

[22] F. Zhang, "Basic results and techniques," in Matrix Theory, Springer, New York, NY, USA, 2011.

[23] D. Cao, "Bounds on eigenvalues and chromatic numbers," Linear Algebra and Its Applications, vol. 270, no. 1-3, pp. 1-13, 1998.

[24] J. H. Smith, "Some properties of the spectrum of a graph," in Combinatorial Structures and Their Applications, pp. 403-406, Gordon and Breach, New York, NY, USA, 1970. 